\title{
Study the One-bit Hard Combination Scheme on Cognitive Radio Users
}

\author{
Md. Shamim Hossain \\ Dept. of Computer Science \& Engineering, Islamic University, Kushtia-7000, Bangladesh \\ shamimmalitha@yahoo.com \\ Mohammad Mamunur Rashid \\ School of Science and Technology, Bangladesh Open University, gazipur-1705, Bangladesh \\ mamunbou@gmail.com \\ Md. Nazrul Islam \\ Dept. of Computer Science \& Engineering, Islamic University, Kushtia-7000, Bangladesh \\ nazrul@cse.iu.ac.bd \\ Md. Abdullah-Al-Mashud \\ Dept. of AECE, Islamic University, Kushtia-7000, Bangladesh \\ ms.mashud@yahoo.com \\ Md. Nurul Islam \\ Dept. of mathematics, Islamic University, Kushtia-7000, Bangladesh \\ nuru_math_iu@yahoo.com
}

\begin{abstract}
This paper describes the performance of onebit hard combination scheme of cooperative spectrum sensing for different number of cognitive radio users. We evaluate cooperative spectrum sensing by simulating OR rule as a hard combination data fusion rule. Energy detector is used to observe the presence of primary user (PU) signal. It improves the probability of detection by collaborating to detect PUs signal in cognitive radio (CR) system. Simulation result shows that the probability of missed detection is decreasing for both conventional hard combination and 1-bit hard combination scheme with OR rule correspondingly with increasing the probability of false alarm. It is shown that 1-bit scheme has much better performance than the conventional hard combination scheme. This is also shown that the probability of missed detection is decreased even though CR user is increased. Here missed detection probability is decreased when the number of user increases.
\end{abstract}

Index Terms - Cognitive Radio, Spectrum Sensing, Data Fusion, Hard Combination

\section{INTRODUCTION}

As the demand for wireless communications increases, the spectrum scarcity has been become a major issue for service providers to deploy new services or enhance the capacity of existing applications. Recently, cognitive radio has been under active consideration to deal with conflict between the spectrum demand and inefficient spectrum utilization $[1,2]$. Cognitive radio $(\mathrm{CR})$ is a promising technique to improve the spectral efficiency of the wireless networks [3].Cognitive users need to detect the presence of licensed users in frequency band to be utilized. The use of a spectrum sensing technique enables to detect spectral holes and opportunistically use underutilized frequency bands. A number of single user spectrum sensing methods have been proposed $[4,5]$. The use of a matched filter can provide optimum performance with prior knowledge on the primary user [4]. Cyclostationary feature detection can detect the signal in very low interference to noise power ratio (INR) condition, but it still requires some prior knowledge on the primary user [4]. Energy detection is simple to implement, but it may suffer from the presence of fading or shadowing [5]. Without prior knowledge on the primary user, the sensing performance of a single user spectrum sensing method may significantly deteriorate in deep fading environments [5]. To overcome this problem, the use of cooperative spectrum sensing has been proposed to achieve so-called multi-user diversity (MUD) gain [6-8]. As one of the most important components of $\mathrm{CR}$, spectrum sensing enables the secondary users to adapt to the environment by detecting the spectrum holes without causing interference to the primary network. Cooperative spectrum sensing has been proposed as a solution to combat the multipath fading or shadowing effects [9-11]. Information from the cooperative secondary users is combined in the fusion center to make the final decision. Traditional one-bit hard decision for cooperative spectrum sensing has been investigated in [12-14], in which secondary users exchange only one-bit decisions rather than the sensing statistics. Soft 
combination in cooperative spectrum sensing has been proposed in [15-16], which outperforms one bit hard decision. However, the soft combination scheme requires much more overhead, e.g. the perfect channel state information between the primary users and secondary users are required for the maximum ratio combination (MRC). Double threshold energy detection has been studied [17]. Li Jiajun et al. proposed the weighted one bit hard combination scheme for cooperative spectrum sensing. Here, we have studied one-bit hard combination scheme for different number of cognitive radio users.

This paper is organized as follows: Section II describes different types of spectrum techniques. Section III provides data fusion technique. Section IV explains the system model under consideration is described. Section V discussed one-bit hard combination scheme. Section VI describes simulation result and discussion. And finally conclusion is drawn in section VII.

\section{Spectrum Sensing Technique}

Spectrum sensing methods for a cognitive radio system can be listed as follows.

\section{A. Matched Filter Detection}

The optimal way for any signal detection is a matched filter [18], since it maximizes received signal-to-noise ratio. However, a matched filter effectively requires demodulation of a primary user signal. This means that cognitive radio has a priori knowledge of primary user signal at both PHY and MAC layers, e.g. modulation type and order, pulse shaping, packet format. Such information might be pre-stored in CR memory, but the cumbersome part is that for demodulation it has to achieve coherency with primary user signal by performing timing and carrier synchronization, even channel equalization. This is still possible since most primary users have pilots, preambles, synchronization words or spreading codes that can be used for coherent detection. For examples: TV signal has narrowband pilot for audio and video carriers; CDMA systems have dedicated spreading codes for pilot and synchronization channels; OFDM packets have preambles for packet acquisition. The main advantage of matched filter is that due to coherency it requires less time to achieve high processing gain since only $O(1 / S N R)$ samples are needed to meet a given probability of detection constraint [19]. However, a significant drawback of a matched filter is that a cognitive radio would need a dedicated receiver for every primary user class.

\section{B. Energy Detection}

One approach to simplify matched filtering approach is to perform non-coherent detection through energy detection. This sub-optimal technique has been extensively used in radiometry. An energy detector can be implemented similar to spectrum analyzer by averaging frequency bins of a Fast Fourier Transform (FFT) [21]. Processing gain is proportional to FFT size $N$ and observation/averaging time $T$. Increasing $N$ improves frequency resolution which helps narrowband signal detection. Also, longer averaging time reduces the noise power thus improves $S N R$. However, due to non-coherent processing $O(1 / S N R 2)$ samples are required to meet a probability of detection constraint [19]. There are several drawbacks of energy detectors that might diminish their simplicity in implementation. First, a threshold used for primary user detection is highly susceptible to unknown or changing noise levels. Even if the threshold would be set adaptively, presence of any in-band interference would confuse the energy detector. Furthermore, in frequency selective fading it is not clear how to set the threshold with respect to channel notches. Second, energy detector does not differentiate between modulated signals, noise and interference. Since, it cannot recognize the interference, it cannot benefit from adaptive signal processing for canceling the interferer. Furthermore, spectrum policy for using the band is constrained only to primary users, so a cognitive user should treat noise and other secondary users differently. Lastly, an energy detector does not work for spread spectrum signals: direct sequence and frequency hopping signals, for which more sophisticated signal processing algorithms, need to be devised. In general, we could increase detector robustness by looking into a primary signal footprint such as modulation type, data rate, or other signal feature.

\section{Cyclostationary Feature Detection}

Modulated signals are in general coupled with sine wave carriers, pulse trains, repeating spreading, hoping sequences, or cyclic prefixes which result in built-in periodicity. Even though the data is a stationary random process, these modulated signals are characterized as cyclostationary, since their statistics, mean and autocorrelation, exhibit periodicity. This periodicity is typically introduced intentionally in the signal format so that a receiver can exploit it for: parameter estimation such as carrier phase, pulse timing, or direction of arrival. This can then be used for detection of a random signal with a particular modulation type in a background of noise and other modulated signals. Common analysis of stationary random signals is based on autocorrelation function and power spectral density. On the other hand, cyclostationary signals exhibit correlation between widely separated spectral components due to spectral redundancy caused by periodicity [20].

\section{Covariance Detection}

This method determines if a primary user is present from the covariance matrix of the received signal, it uses the property that the off diagonal elements of the covariance matrix is non-zero when a primary user is present and zero otherwise.

\section{E. Wavelet Detection}

The spectrum of interest is decomposed as a train of consecutive frequency sub bands, wavelet transform is used to detect the irregularities in these bands (PSD is relatively smooth within the sub bands and possess irregularities at the edges between two neighboring sub bands). Wavelet transform carries information about the 
locations of these frequencies and the PSD of the sub bands. Vacant frequency bands are obtained by detecting the singularities of the PSD of the signal observed, by taking the wavelet transform of its PSD.

\section{F. Cooperative Sensing}

Cooperation is proposed as a solution to problems that arise in spectrum sensing due to noise uncertainty, fading, and shadowing. Cooperative sensing decreases the probabilities of miss-detection and false alarm considerably. In addition, cooperation can solve hidden primary user problem and it can decrease sensing time. In this technique, the cognitive radio users are populated in the range of primary transmitter to perform its individual signal detection using some detection methods and determine the reliability of its own detection results. We adopt OR rule of hard decision fusion method for this evaluation. Where, users send a binary local decision to data fusion center.

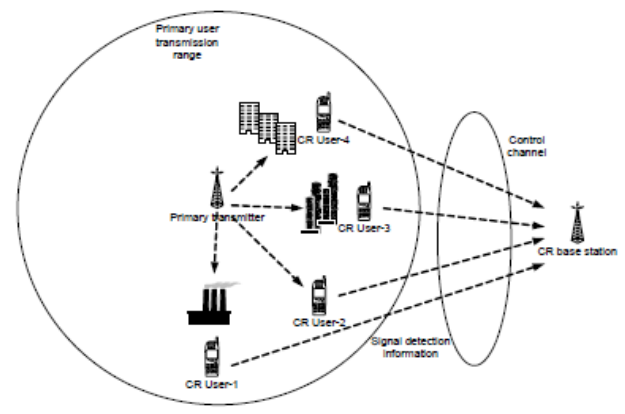

Figure 1: Cooperative signal detection model

Figure 1 shows system model of cooperative signal detection where only one cognitive radio user could be able to detect the primary signal. The other cognitive radio users are not able to distinguish existence of the primary signal by fading and shadowing effect. The users are populated in the range of primary transmitter. Under this condition, it is expected that it can improve the signal detection probability. Collaboration among cognitive radio users is theoretically more accurate and convenient.

\section{DATA FUSION}

In cooperative sensing, data fusion is a process of combining local sensing data for hypothesis testing, which is also an element of cooperative sensing in [22]. In hard combination scheme CR users make a local decision and transmit the one bit decision for hard combining. In order to realize the cooperative detection among CR users, the spectrum sensing and signal detection information over individual users should be sent to a fusion center for further process and the fusion center makes the final decision whether primary user signal is present or absent.

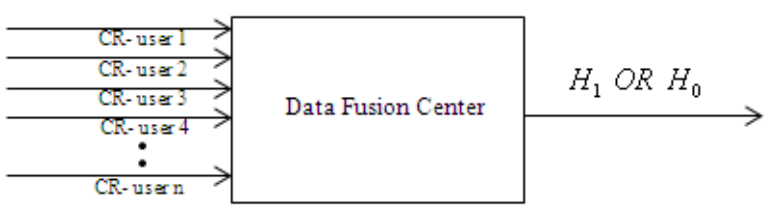

Figure 2: Data fusion center.

As described in Figure 2, information of local signal observation from all cognitive users transmits to data fusion center. They forward 1-bit local detection to avoid communication overhead when CR users increased. Then, the final decision is performed whether signal is present $\left(H_{1}\right)$ or absent $\left(H_{0}\right)$ by regarding to decision rule. With a hard decision counting rule, the fusion center implements an $n$-out-of- $M$ rule that decides on the signal present hypothesis whenever at least $n$ out of the $M$ local decisions indicate $H_{1}$. Assuming uncorrelated decisions, the probability of detection at the fusion center is given by [23]:

$P_{d}=\sum_{n}^{M}\left(\begin{array}{c}M \\ k\end{array}\right) P_{d, i}{ }^{k}\left(1-P_{d, i}\right)^{M-k}$

Where, $P_{d, i}$ is the probability of detection node. In OR rule, if any one of the local decisions sent to the decision maker is a logical one (i.e., any one of the cognitive radio user decides that the signal of interest is present), the final decision made by the decision maker is one (i.e., decision maker decides that the signal of interest is present). Cooperative detection performance with this fusion rule can be evaluated by setting $n=1$ in equation (1).

$$
P_{d, O R}=1-\left(1-P_{d, i}\right)^{M}
$$

\section{SYSTEM MODEL}

The energy detector consists of a square law device followed by a finite time integrator (fig.3). The output of the integrator at any time is the energy of the input to the squaring device over the interval $T$ in the past. The noise pre-filter serves to limit the noise bandwidth; the noise at the input to the squaring device has a bandlimited, flat spectral density in [23].

The binary hypothesis test for spectrum sensing at the nth time instant is formulated as [8]:

$$
\begin{aligned}
& H_{0}: r(n)=w(n) \\
& H_{1}: r(n)=x(n)+w(n)
\end{aligned}
$$

Where $r(n)$ is the signal to be detected, $x(n)$ is the primary signal with power $\sigma_{x}{ }^{2}$, and $w(n)$ is the complex additive white Gaussian noise (AWGN) with zero-mean and variance $\sigma_{n}^{2}$. 


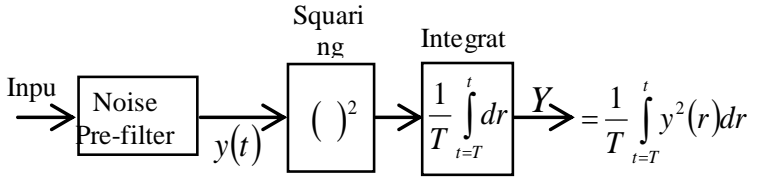

Figure 3: Energy Detection

Without loss of generality, it is assumed that all the powers of the local noise are the same and $\sigma_{n}{ }^{2}$ is normalized to be 1 .

$H_{0}$ and $H_{1}$ denote the hypotheses corresponding to the absence and presence of the primary signal respectively. The local decision statistic is given by

$$
S=\sum_{n=1}^{M}|r(n)|^{2}
$$

Where, $\mathrm{M}$ is the number of samples. When there is only noise present,

$$
S_{\mathrm{o}}=\sum_{n=1}^{M}|w(n)|^{2}
$$

Then the probability of false alarm can be expressed as [25-30]

$$
P_{f}=P\left(S_{0}>\lambda\right)=\frac{\Gamma\left(\frac{M}{2}, \frac{\lambda}{2}\right)}{\Gamma\left(\frac{M}{2}\right)}
$$

Where $\Gamma(\cdot, \cdot)$ is the incomplete gamma function, $\Gamma(\cdot)$ is the gamma function and $\lambda$ is the final threshold of the local detector to decide whether there is a primary user present. When there is primary signal present,

$$
S_{1}=\sum_{n=1}^{M}|x(n)+w(n)|^{2}
$$

The probability of detection can be expressed as [26-32]

$$
P_{d}=P\left(S_{1}>\lambda\right)=\frac{Q_{M}}{2}(\sqrt{2 M \gamma, \sqrt{\lambda}})
$$

Where, $\gamma=\frac{\sigma_{x}^{2}}{2 \sigma_{n}{ }^{2}}=\frac{\sigma_{x}^{2}}{2}$ denotes the signal to signal to noise ratio (SNR),

$$
Q_{m}(a, b)=\int^{\infty} x(x / a)^{m-1} e^{-\left(x^{2}+a^{2}\right) / 2} . I_{m-1}(a x) d x
$$

is the generalized Marcum's Q function and $I_{m-1}$ denotes the modified Bessel function of the first kind.
Suppose there are $N$ secondary users to perform cooperative spectrum sensing. As shown in Fig 4, two thresholds $\left(\lambda_{1}, \lambda_{2}\right)$ are designed for one-bit hard combination of the proposed cooperative spectrum sensing, which divides the whole range of the decision statistic of the local spectrum sensing into three regions.

$$
\begin{array}{c|ll}
\operatorname{Energy}(Y) & & \\
\lambda_{2} & \text { Region 2, } & \omega_{2}=1 \\
\cline { 2 - 3 } \lambda_{1} & \text { Region 1, } & \omega_{1}=1 \\
\cline { 2 - 3 } & \text { Region 0, } & \omega_{0}=0
\end{array}
$$

Figure 4: Cooperative spectrums sensing with two thresholds

The following weights are adopted in our scheme $\omega_{0}=0, \omega_{1}, \omega_{2}=1$. In the fusion center, if the energy value falls into the corresponding region, it will be judged as "1", no information or "0". And the decision rule adopted is given

$$
D=\left\{\begin{array}{cc}
1 & N_{2}+N_{1} \omega_{1} \geq 1 \\
0 & \text { otherwise }
\end{array}\right.
$$

Where $N_{1}$ is the number of local detectors the energies of which are greater than $\lambda_{1}$ and less than $\lambda_{2}, N_{2}$ is the number of local detectors the energies of which are greater than $\lambda_{2}$. Obviously, $\omega_{1}$ should be more than $1 / N_{1}$ and less than 1 . Let $Q_{f 2}$ be the probability that one or more observation local energy values are greater than $\lambda_{2}$ and $Q_{f 1}$ be the probability that more than $N_{1}=\left\lfloor 1 / \omega_{1}\right\rfloor$ observation local energy values are greater than $\lambda_{1}$ and less than $\lambda_{2}$. Here, $\lfloor x\rfloor$ is the largest integer that is no more than $x$. We have from [21]

$$
Q_{f 2}=1-P\left(N_{2}=0 \mid H_{0}\right)=1-\left(1-P_{f 2}\right)^{N}
$$

$Q_{f 1}=P\left(N_{2}=0 \mid H_{0}\right) \cdot \sum_{J=N_{1}}^{N} P\left(N_{1}=j \mid H_{0}\right)$

$=\left(1-P_{f 2}\right)^{N} \cdot \sum_{j=N_{1}}^{N}\left(\begin{array}{c}N \\ j\end{array}\right)\left(P_{f 1}-P_{f 2}\right)^{j} \cdot\left(1-P_{f 1}+P_{f 2}\right)^{N-j}$

Where $P_{f n}$ is the probability that the local energy value is greater than $\lambda_{n}$ and according to (4)

\section{ONE BIT HARD COMBINATION SCHEME}




$$
P_{f n}=P\left(S_{0}>\lambda_{n}\right)=\frac{\Gamma\left(\frac{M}{2}, \frac{\lambda_{n}}{2}\right)}{\frac{M}{2}}
$$

We have

$$
\lambda_{n}=2 \Gamma^{-1}\left(\frac{M}{2}, P_{f n} \Gamma\left(\frac{M}{2}\right)\right)
$$

$\Gamma^{-1}(\cdot, \cdot)$ denotes the inverse of the incomplete gamma function. Then the probability of false alarm in the fusion center can be expressed as

$$
Q_{f}=Q_{f 1}+Q_{f 2}
$$

Suppose all the channels between the primary user and secondary users are independent identically distributed. The probability of detection in the fusion center can be expressed as in [21]

$$
\begin{gathered}
Q_{d}=1-\left(1-P_{d 2}\right)^{N}+\left(1-P_{d 2}\right)^{N} \\
\sum_{j=N_{1}}^{N}\left(\begin{array}{c}
N \\
j
\end{array}\right)\left(P_{d 1}-P_{d 2}\right)^{j} \cdot\left(1-P_{d 1}+P_{d 2}\right)^{N-j}
\end{gathered}
$$

Where $P_{d n}$ is the probability that the local energy value is greater than $\lambda_{n}$ when there is primary present according to (6) and (7).

\section{SimULATION RESULT}

Simulation was done on MATLAB version R2011a under AWGN channel. We use complementary receiver characteristics (ROC) analysis for the signal detection theory to study the performance of one-bit hard combination scheme of cooperative spectrum sensing for different number of cognitive Radio users. Complementary ROC has been widely used in the signal detection theory due to the fact that it is an ideal technique to quantify the tradeoff between the probability of detection and the probability of false alarm. The following simulation was done by taking time bandwidth factor $\mathrm{u}=\mathrm{TW}=1000$, primary user transmitting signal's samples $=2000$ and probability of false alarm (Pfa) is used from 0.01 to 1 by increasing 0.01 , where $100 \mathrm{Pfa}$ is used.

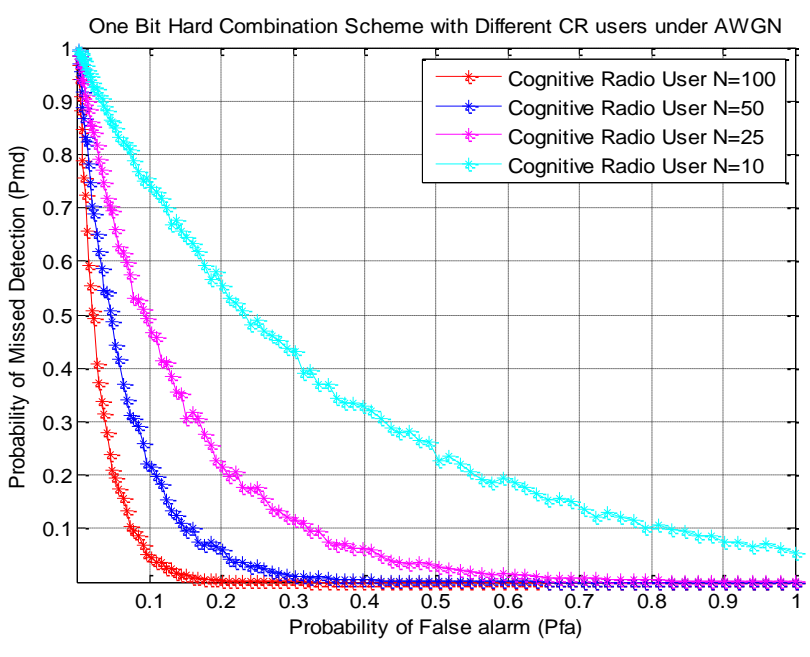

Figure 5: One bit hard combination scheme versus conventional scheme with 10 user

Figure 5 shows complementary ROC of conventional OR rule and 1-bit OR rule in the hard combination scheme. Where $10 \mathrm{CR}$ users and $\mathrm{SNR}=15 \mathrm{~dB}$ were taken. It shows that the probability of missed detection is decreasing for both conventional hard combination and 1bit hard combination scheme with OR rule correspondingly with increasing the probability of false alarm. It also shows that probability of missed detection in 1-bit hard combination scheme is reduced significantly than the conventional hard combination scheme although same CR user is used. 1-bit hard combination scheme has much better performance than conventional one-bit scheme.

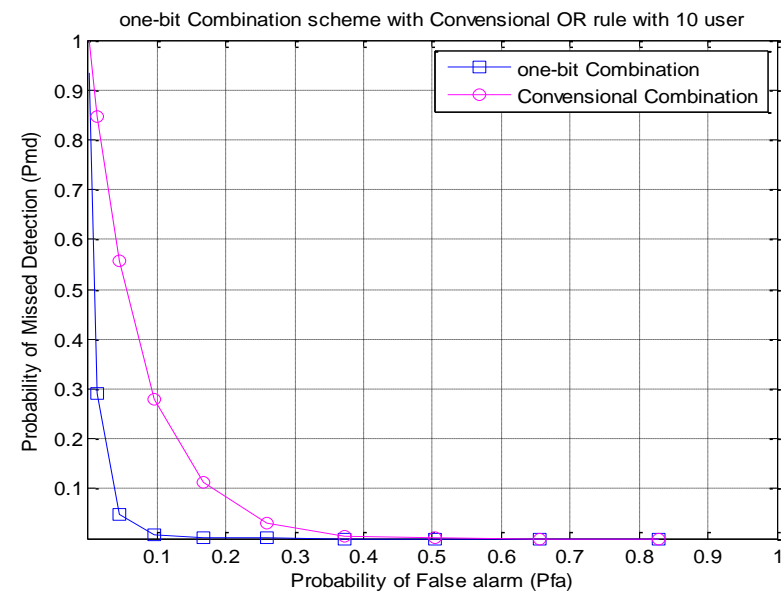

Figure 6: One bit hard combination for different number CR users

Figure 6 shows one bit hard combination scheme for different number of cognitive radio users. It is considered that the probability of missed detection versus probability of false alarm under AWGN channel with the complementary ROC of cooperative spectrum sensing with $\mathrm{OR}$ rule \& different $\mathrm{CR}$. The single to noise ratio was taken $\mathrm{SNR}=15 \mathrm{~dB}$. This simulation is tested by taking cognitive radio user $\mathrm{N}=10,25,50$ and 100 . Simulation result shows that with the increasing the probability of false alarm from 0.01 to 1 , the missed detection is 
decreasing. It also shows that probability of missed detection is decreasing when the CR user increases. When we take CR user 100 then missed detection probability is decreased than the $\mathrm{CR}$ user 10. So cooperation with more number of $\mathrm{CR}$ users in one-bit hard combination scheme, spectrum sensing is evaluated better performance.

\section{CONCLUSIONS}

Cognitive radio improves the utilization efficiency of the radio spectrum. Cooperative communications can play a key role in the development of CR networks. Simulation result demonstrated that the probability of missed detection is decreasing for both conventional hard combination and 1-bit hard combination scheme with OR rule correspondingly with increasing the probability of false alarm. It is shown that 1-bit scheme has much better performance than the conventional hard combination scheme. We saw that the probability of missed detection is decreased on increasing users.

\section{REFERENCES}

[1] A. Ghasemi and E.S. Sousa, "Opportunistic spectrum access in fading channels through collaborative sensing," IEEE Journal of Commun., vol. 2, no. 2, pp. 1932-1940, Mar. 2007.

[2] G. Ganesan and Y. G. Li, "Cooperative spectrum sensing in cognitive radio, part 1: two user networks," IEEE Trans. Wireless Commun., vol. 6, no. 6, pp. 1536-1544, June 2007.

[3] HAYKIN S. Cognitive Radio: Brain-empowered Wireless Communications, Selected Areas in Communications [J]. IEEE Journal on, 2005, 23(2): 201-220.

[4] D. Cabric, S. M. Mishra, and R. W. Brodersen, "Implementation issues in spectrum sensing for cognitive radios," in Proc. Asilomar Conf. Signals, Systems and Computers, pp. 7-10, Nov. 2004.

[5] V. I. Kostylev, "Energy detection of a signal with random amplitude," in Proc. IEEE Int. Conf. Commun. pp. 1606-1610, May 2002.

[6] A. Ghasemi and E. S. Sousa, "Spectrum sensing in cognitive radio networks: the cooperationprocessing tradeoff," Wireless Commun. and Mobile Comput., vol. 7, no. 1, pp. 1049-1060, Sept. 2007.

[7] C. Sun, W. Zhang, and K. B. Letaief, "Cooperative spectrum sensing for cognitive radios under bandwidth constraints," in Proc. IEEE

[8] Shahoreare, Shamim Hossain, Ibrahim Abdullah and Alamgir Hossain, "Cooperative Spectrum Sensing over Rayleigh Fading Channel in Cognitive Radio", pp. 2583- 2592, IJECSE, Volume1, Number 4, ISSN 2277-1956/V1N4-2583-2592.

[9] GANESAN G, LI Ye. Cooperative Spectrum Sensing in Cognitive Radio Networks[C]// Proceedings of DySPAN 2005. Baltimore, Maryland USA: IEEE Press, 2005: 137-143.
[10] GHASEMI A, SOUSA E S. Collaborative Spectrum Sensing for Opportunistic Access in Fading Environments Proceedings of DySPAN 2005. Baltimore, Maryland USA: IEEE Press, 2005: 131136.

[11] RENZO M Di, GRAZIOSI F, SANYUCCI F. Cooperative Spectrum Sensing in Cognitive Radio Networks over Correlated Log-Normal Shadowing [C]// Proceedings of VTC 2009-Spring. Barcelona, Spain: IEEE Press, 2009: 1-5.

[12] GHASEMI A, SOUSA E S. Collaborative Spectrum Sensing for Opportunistic Access in Fading Environments Proceedings of DySPAN 2005. Baltimore, Maryland USA: IEEE Press, 2005: 131136.

[13] RENZO M Di, GRAZIOSI F, SANYUCCI F. Cooperative Spectrum Sensing in Cognitive Radio Networks over Correlated Log-Normal Shadowing [C]// Proceedings of VTC 2009-Spring. Barcelona, Spain: IEEE Press, 2009: 1-5.

[14] MISHRA S M, SAHAI A, BRODERSEN R W. Cooperative Sensing among Cognitive Radios [C]// Proceedings of ICC'06. Istanbul, Turkey: IEEE Press, 2006: 1658-1663.

[15] YUAN Y, ANXIN L, KAYAMA H. Study on Soft Decision Based Cooperative Sensing in Cognitive Radio Network [C]// Proceedings of VTC 2009-Fall. Anchorage, Alaska USA: IEEE Press, 2009: 1-5.

[16] MA Jun, ZHAO Guodong, LI Ye. Soft Combination and Detection for Cooperative Spectrum Sensing in Cognitive Radio Networks [J]. Wireless Communications, IEEE Transactions on, 2008, 17(11): 4502-4507.

[17] DIGHAN F F, ALOUINI M S, and SIMON M K. On the Energy Detection of Unknown Signals over Fading Channels [C]// Proceedings of ICC'03, Anchorage, Alaska USA: IEEE Press, 2003: 35753579.

[18] J. Proakis, Digital Communications, 3rd edition, Mc Graw Hill

[19] A. Sahai, N. Hoven, R. Tandra, "Some Fundamental Limits on Cognitive Radio", Proc. of Allerton Conference, Monticello, Oct 2004.

[20] W.A.Gardner, "Signal Interception: A Unifying Theoretical Framework for Feature Detection", IEEE Trans. on Communications, vol. 36, no. 8.August 1988

[21] A. V. Oppenheim, R. W. Schafer and J. R. Buck, Discrete-Tme Signal Processing, Prentice Hall, 1999.

[22] Li Jiajun, Tan Zhenhui, Ai Bo1, Yang Shan, "Weighted Hard Combination for Cooperative Spectrum Sensing in Cognitive Radio Networks", research paper, 2011.

[23] I. F. Akyildiz, B. F. Lo, and R. Balakrishnan, "Cooperative Spectrum Sensing in Cognitive Radio Networks: A Survey," Physical Communication (Elsevier) Journal, vol. 4, no. 1, pp. 40-62, March 2011. 
[24] S. Kyperountas, N. Correal, Q. Shi and Zhuan Ye, "Performance analysis of cooperative spectrum sensing in Suzuki fading channels," in Proc. of IEEE Intern. Con. on Cognitive Radio Oriented Wireless Networks and Communications (CrownCom'07), pp. 428-432, June 2008.

[25] H. Urkowitz, "Energy detection of unknown deterministic signals,” Proc. IEEE, vol. 55, pp. 523531, Apr. 1967.

[26] S. Nallagonda, S. Suraparaju, S. D. Roy and S. Kundu, "Performance of energy detection based spectrum sensing in fading channels", in Proc. of IEEE International Conference on Computer and Communication Technology (ICCCT'11), September, pp. 575-580.

[27]F. F. Digham, M. -S. Alouini, and M. K. Simon, "On the energy detection of unknown signals over fading channels," in Proc. IEEE Int. Conf. on Commun., May 2003, vol. 5, pp. 3575-3579.

[28] A. Ghasemi and E. S. Sousa, "Impact of user collaboration on the performance of opportunistic spectrum access," in Proc. IEEE Vehicular Technology Conference (VTC Fall'06), Montreal, September 2006.

[29] S. H Lee, Y. H. Lee, S, "Hard Decision Combiningbased Cooperative Spectrum Sensing in Cognitive Radio Systems".

[30] S. Kyperountas, N. Correal and Q. Shi, "A comparison of Fusion Rules for Cooperative Spectrum Sensing in Fading Channels", EMS Research, Motorola.

[31]N. Armi, N.M. Saad and M. Arshad, "Hard Decision Fusion based Cooperative Spectrum Sensing in Cognitive Radio System", ITB, vol.3, No.2, 2009, pp. 109-122.

[32] S. P. Herath, N. Rajatheva, and C. Tellambura, "Unified Approach for Energy Detection of Unknown Deterministic Signal in Cognitive Radio Over Fading Channels", In: IEEE Inter. Con. on Commun. Workshops, 2009. ICC Workshops 2009. 14-18 June 2009 Page(s): $1-5$.

\section{Authors' Profiles}

Md. Shamim Hossain has been received Bachelor's and Master's degree in Computer Science and Engineering from Islamic University, Kushtia-7003, Bangladesh. Currently he is a Lecturer of the Department of CSE, Islamic University, Kushtia. His research areas of interest include wireless communication, WSN \& Cognitive Radio. His work has produced 13 peer-reviewed scientific International Journal papers.

Mohammad Mamunur Rashid has been received B.Sc. and M.Sc. in Computer Science degree from University of Madras, Chennai, India. Currently he is working as a lecturer in the school of science and technology, Bangladesh Open University. His areas of interest include database management system, wireless communication and cognitive radio.

Md. Nazrul Islam has been received the Bachelor's, Master's degree in computer science and engineering from Madras University, Chennai, India. Currently he is an assistant professor of the department of CSE, Islamic University, Kushtia-7003, Bangladesh. His areas of interest include wireless communication, WSN \& Cognitive Radio.

Md. Abdullah-Al-Mashud has been received the B.Sc (Hons) degree and M.Sc degree in APECE from Islamic University, Kushtia-7003, Bangladesh in 2003 and 2004 respectively. He works as a lecturer in the department of APECE, Islamic University, Bangladesh. His current interest is microprocessor / microcontroller applications in control, automation, medical instruments, environmental monitoring, low cost electronic systems, Medical Image Processing. His work has produced 08 peer-reviewed scientific International and National Journal papers. He has published 04 papers in National and International Conferences.

Md. Nurul Islam has been received the B.Sc. and M.Sc. degree in mathematics from Jahangirnagar University, Savar, Dhaka. Currently he is a lecturer of the department of Mathematics, Islamic University, Kushtia-7003, Bangladesh. His areas of interest include Image Processing, Fluid Mechanics, Numerical Analysis \& wireless communication. 